\title{
Simulation Result of Gallium Indium Nitride Solar Cells
}

\author{
Jin $\mathrm{Wu}$ \\ Changchun University of Science and Technology, Wexin, Changchun, China.
}

InGaN can reach all values of bandgap from 3.42 to $0.7 \mathrm{eV}$, which covers almost the entire solar spectrum. This study is to understand the influence of each parameter of the solar cell for an improved optimization of performance. The yield obtained for a reference cell is $12.2 \%$ for optimal values of doping of the layers. For generation and recombination, performance of the cell varies with these settings. III nitrides have a high absorption coefficient, a very thin layers of material are sufficient to absorb most of the light.

\section{Introduction}

III-N semiconductors are interested materials with a high thermal conductivity and a high melting point, and a direct band gap. They currently represent ideal materials for the development of light emittingdiodes (LEDs) operating in the visible, UV ranges, and laser diodes etc [1-5]. The band gap of these ternary or quaternary materials can be adjusted to absorb the light from infrared to ultraviolet makes these materials very useful for other applications such as photovoltaic and photodiode applications because of the possibility of achieving high efficiency [6-8]. Additionally, III-Nitrides also exhibit other interesting photovoltaic properties such as low effective masses of charge carriers, large absorption coefficients, and high mobility as compared to other materials such as GaAs [9-13]. III-V nitrides has demonstrated its ability to demonstrate its ability for applying in optoelectronic devices, which confirms its potential for high efficiency photovoltaics. [14].

\section{Properties of InGaN used in the simulation}

In this paper, we used PCID software to simulate the model, a freely available for public use [15-16]. The alloy used for simulation consists of $\operatorname{In}_{0.4} \mathrm{Ga}_{0.6} \mathrm{~N}$, which has a bandgap of $2.1 \mathrm{eV}$ and $1 \mathrm{~mm} 2$ cell was

chosen in area for the $\mathrm{p}$-i-n homojunction device and was n-type doping of $1 \times 10^{18} / \mathrm{cm}^{3}$. The InGaN dielectric constant, the intrinsic concentration et $300 \mathrm{~K}$ were fixed at 9.52 and $90.9 \mathrm{~cm}^{-3}$, respectively. For carriers mobility the simulator offers two choices to the user, the first one is the carrier constant mobility, while the second considers mobility through its internal model at $300 \mathrm{~K}$.

\section{Simulation results}

Results are obtained using the file excitation (1 Sun) with PC1D software and the characteristics of I-V and the power of the cell are represented by Fig. 1 and Fig. 2 below:

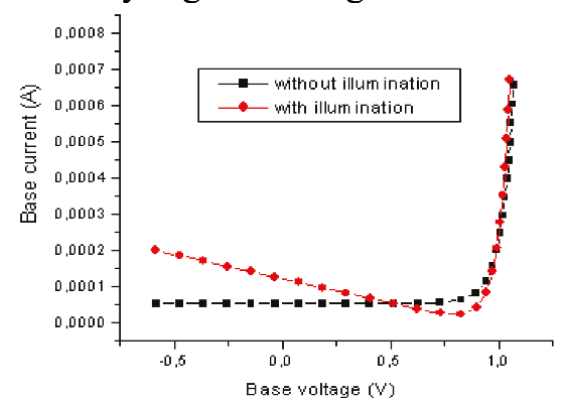

Fig. 1. I-V characteristic of the reference cell 


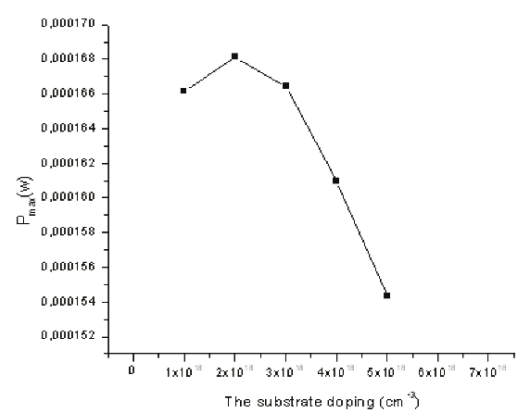

Fig. 2. The maximum power of the cell as a function of substrate doping for n-substrate $\mathrm{p}$-InGaN.

From Figure Fig. 2, both carriers lifetime mobility degrades while the Shockley-Read-Hall and Auger recombination rates increase. This causes a significant decrease in performance for a heavily doped substrate. III-V nitrides show a very low diffusion length due to the high dislocation density in currently available materials, especially those relevant to this work where high In \% InGaN layers are required. But, III-nitrides have a high absorption coefficient thus requiring very thin layers of material to absorb most of the incoming photons.

Conclusion:

InGaN based solar cells have a low diffusion length may due to the high dislocation density in present materials with the highest reported minority carrier lifetime. Thus, the materials issues are resolved and lower dislocation InGaN layers become available and III-V nitrides an important candidate in the development of high efficiency photovoltaic and other important devices.

\section{References:}

[1] Hiroshi Amano, Masahiro Kito, Kazumasa Hiramatsu and Isamu Akasaki, Japanese Journal of Applied Physics, (1989) 28, L2112 https://doi.org/10.1143/JJAP.28.L2112

[2] Hiroshi Amano, Tsunemori Asahi and Isamu Akasaki, Japanese Journal of Applied Physics, (1990) 29, L205 https://doi.org/10.1143/JJAP.29.L205

[3] BT Tran, Noritoshi Maeda, Masafumi Jo, Daishi Inoue, Tomoka Kikitsu \& Hideki

Hirayama, Scientific Reports (2016) 6, 35681 https://doi.org/10.1038/srep35681

[4] B Tran, Chen-Hauw Ming, Kung Liang Lin, Hao-Ming Chen, Ching Chian Wang, Chien Chih Chen, Chih Yung Huang, Chen Chen Chung and Edward Yi Chang, ECS

Transactions, (2013) 50, 1 https://doi.org/10.1149/05048.0001ecst

[5] Maocheng Shan, Yi Zhang,Tinh B. Tran, Jie'an Jiang, Hanling Long, Zhihua Zheng, An'ge Wang, Wei Guo, Jichun Ye, Changqing Chen, Jiangnan Dai, and Xiaohang Li, ACS Photonics (2019) 6, 2387-2391 https://doi.org/10.1021/acsphotonics.9b00882

[6] S.M. Sze and K. K. Ng, 'Physics of Semiconductor Devices', Third Edition, John Wiley and Sons, Interscience, 2006.

[7] B-Tran, Edward-Yi Chang, Hai-Dang Trinh, Ching-Ting Lee, Kartika Chandra Sahoo, Kung-Liang Lin, Man-Chi Huang, Hung-Wei Yu, Tien-Tung Luong, Chen-Chen Chung, Chi- Lang Nguyen, Solar Energy Materials and Solar Cells (2012) 102, 208-211 https://doi.org/10.1016/j.solmat.2012.03.030

[8] Franky Lumbantoruan, Yuan-Yee Wong, Yue-Han Wu, Wei-Ching Huang, Niraj Man Shrestra, Tung Tien Luong, Tran Binh Tinh, Edward Yi Chang, 2014 IEEE International Conference on Semiconductor Electronics (ICSE2014) https://doi.org/10.1109/SMELEC.2014.6920785 
[9] Chen-Chen Chung, Hsiao-Chieh Lo, Yen-Ku Lin, Hung-Wei Yu, Binh Tinh Tran, KungLiang Lin, Yung Chang Chen, Nguyen-Hong Quan, Edward Yi Chang, Yuan-Chieh Tseng, Mater. Res. Express (2015) 2, 055505 https://doi.org/10.1088/2053-1591/2/5/055505 [10] Hung Wei Yu, Chen Chen Chung, Chin Te Wang, Hong Quan Nguyen, Binh Tinh Tran, Kung Liang Lin, Chang Fu Dee, Burhanuddin Yeop Majlis, Edward Yi Chang, Jpn. J. Appl. Phys. (2012) 51, 080208 https://doi.org/10.1143/JJAP.51.080208

[11]. Chen-Chen Chung, Binh Tinh Tran, Hau-Vei Han, Yen-Teng Ho, Hung-Wei Yu, KungLiang Lin, Hong-Quan Nguyen, Peichen Yu, Hao-Chung Kuo, Edward Yi Chang, Electronic Materials Letters (2014) 10, 457-460 (2014) https://doi.org/10.1007/s13391-013-3202-3

[12] Chen-Chen Chung, Binh Tinh Tran, Ming-Hung Han, Kung-Liang Lin, Hung-Wei Yu, YenTeng Ho, Chun-Yen Chang, Edward Yi Chang, Electronic Materials Letters 967 https://doi.org/10.1007/s13391-014-3201-Z

[13] Hong-Quan Nguyen, Edward Yi Chang, Hung-Wei Yu, Hai-Dang Trinh, Chang-Fu Dee, Yuen-Yee Wong, Ching-Hsiang Hsu, Binh-Tinh Tran, Chen-Chen Chung, Express (2012) 5 055503 https://doi.org/10.1143/APEX.5.055503

[14] Henry Mathieu. Physique des semi-conducteurs et des composants électroniques, Masson, (1987).

[15] P. A. Basore and D. A. Clugston, PC1d Version 5.9, Sydney, Australia: Univ. New South Wales, 2003.

[16] www.pv.unsw.edu.au/info-about/our-school/products-services/pcld 